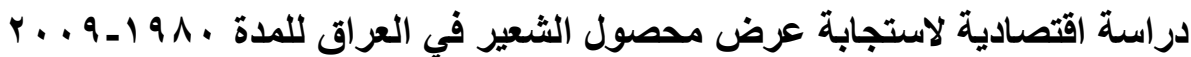

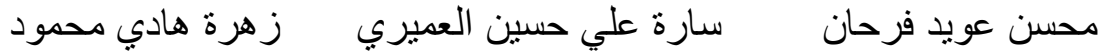

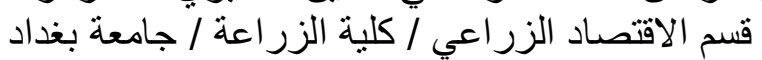

\section{الخلاصة}

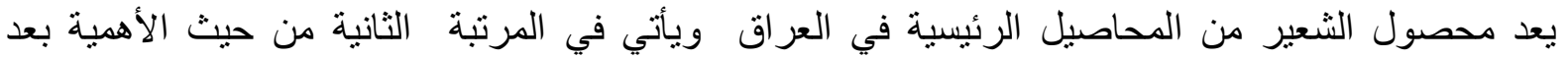

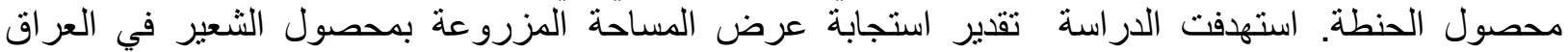

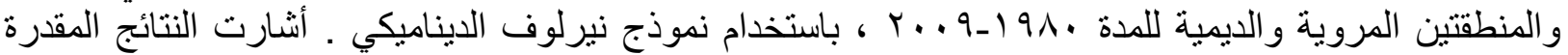

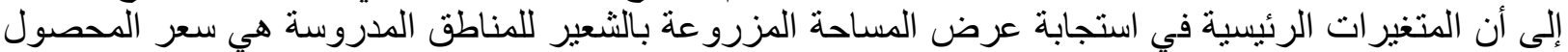

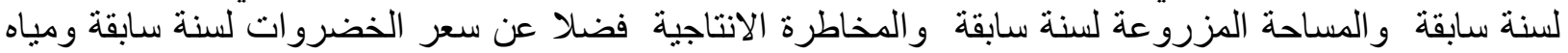

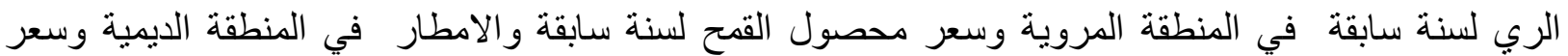

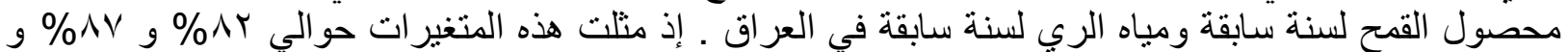

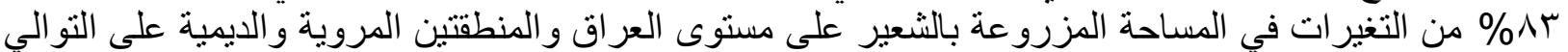

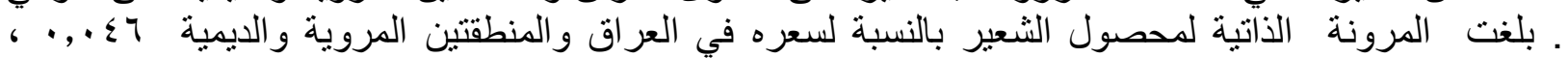

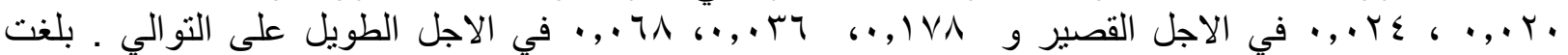

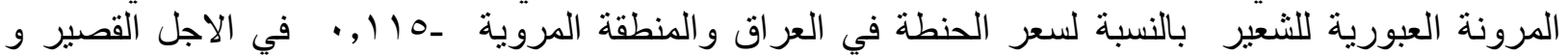

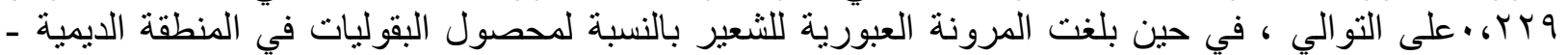

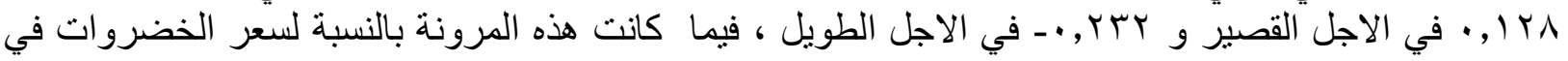

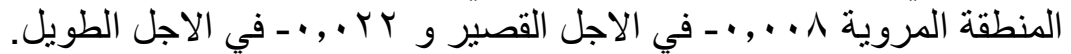

\section{المقدمة}

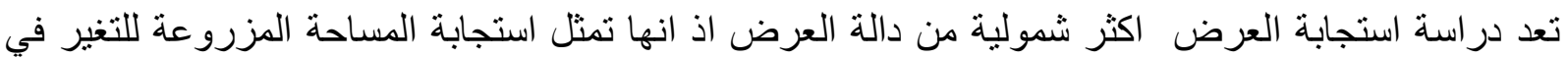

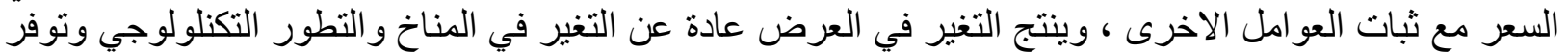

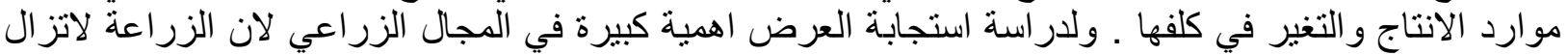

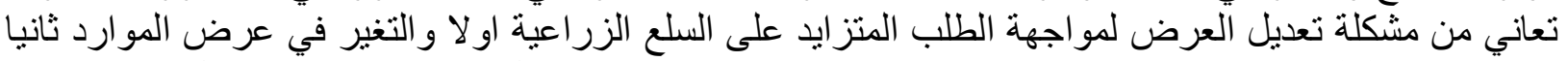

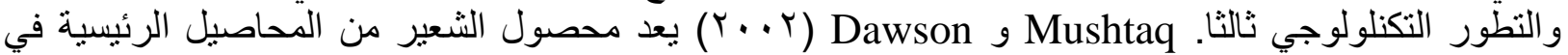

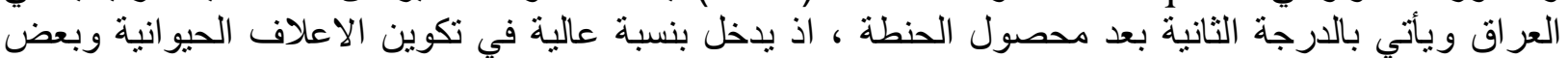

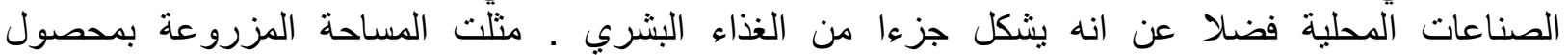

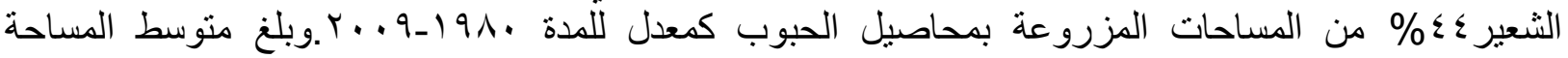

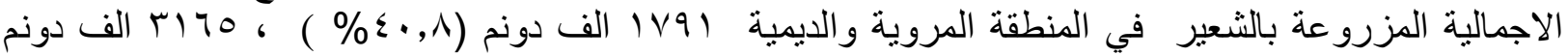

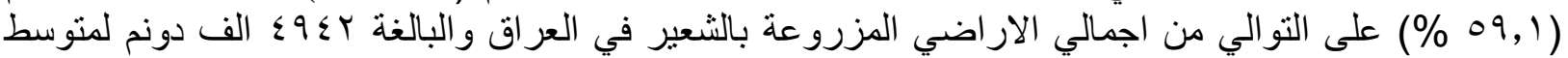

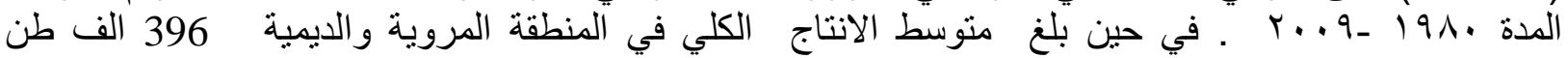

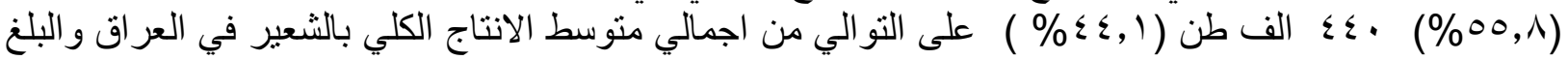

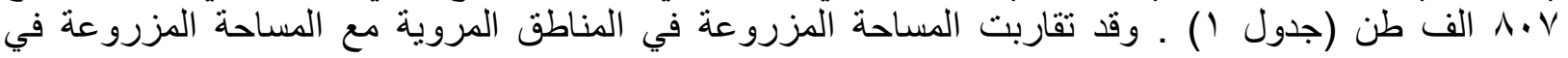

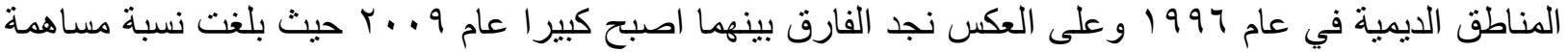

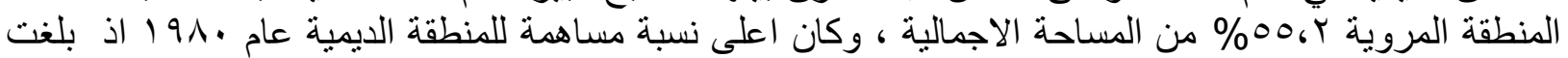

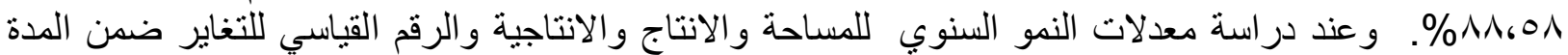

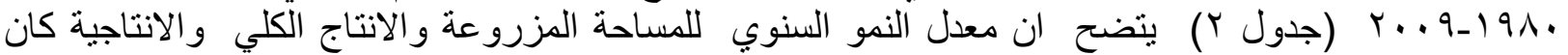

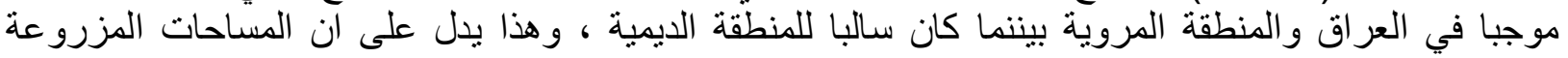

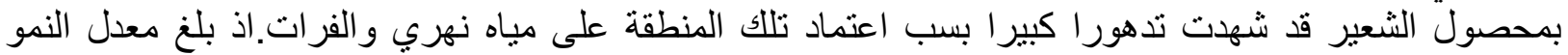

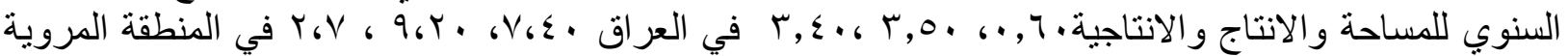

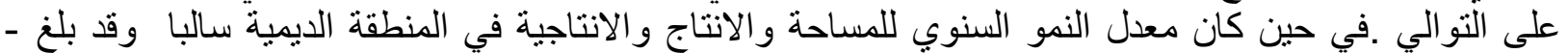

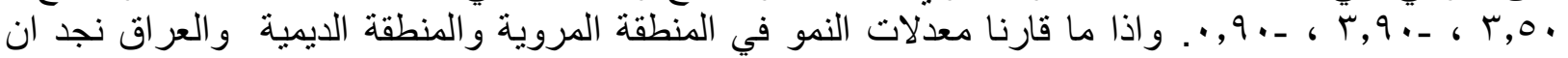

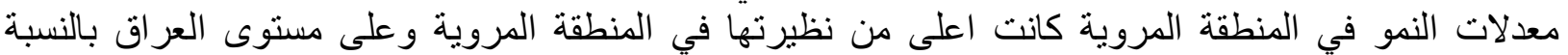

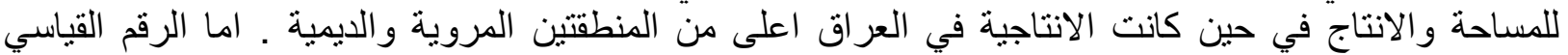

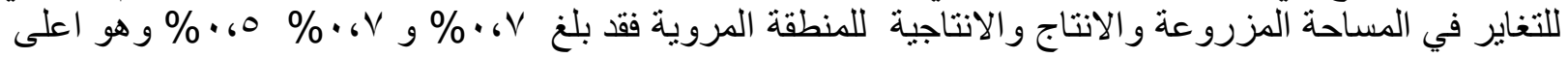




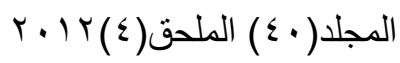 \\ ISSN:2224-9796(Online) \\ ISSN: 1815-316X(Print)}

مجلة زر اعة الر افدين

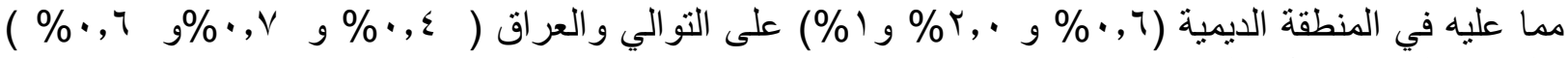

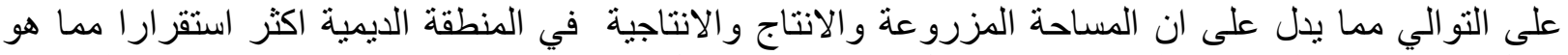

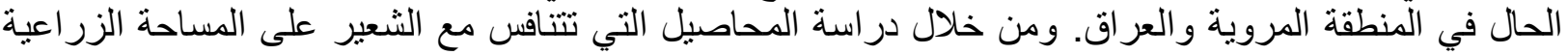

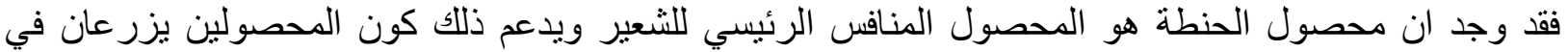

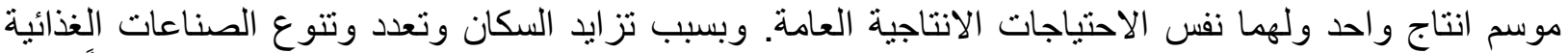

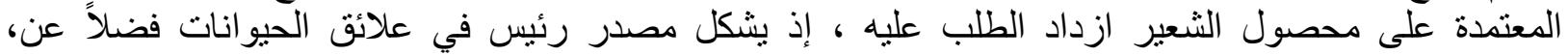

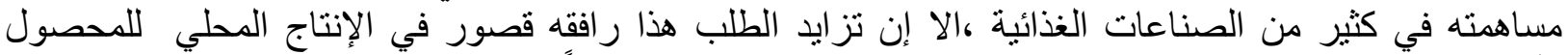

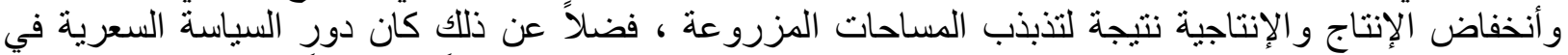

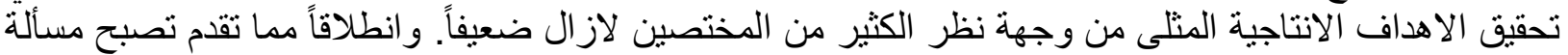

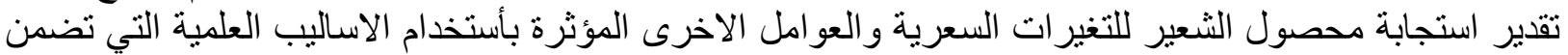

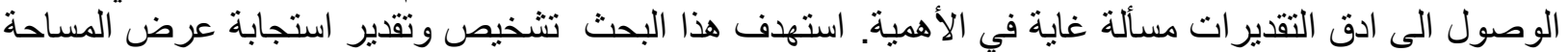

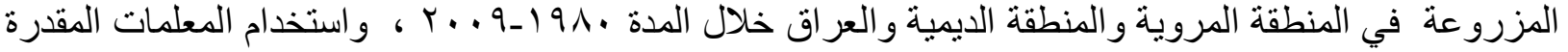

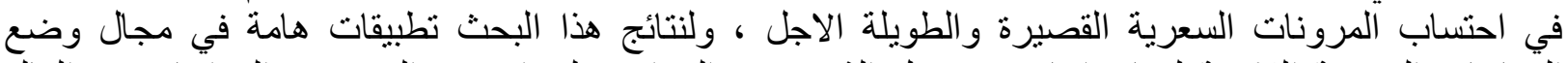

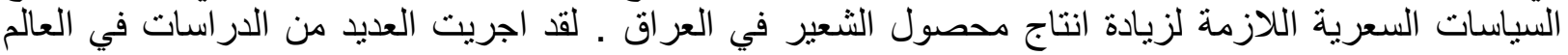

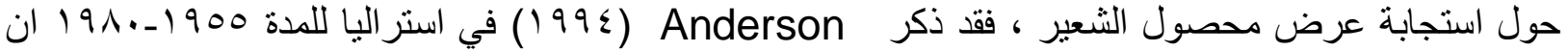

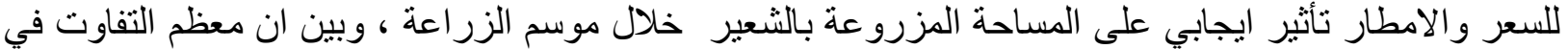

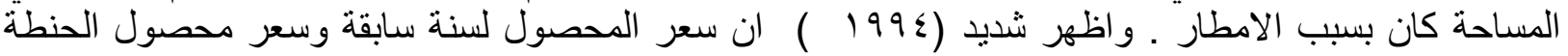

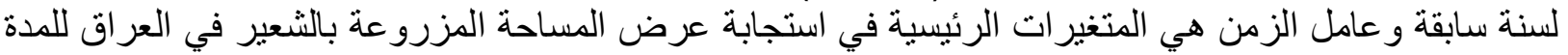

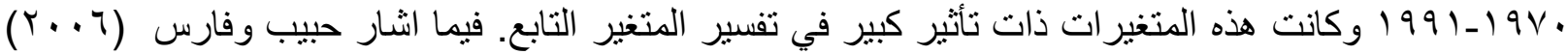

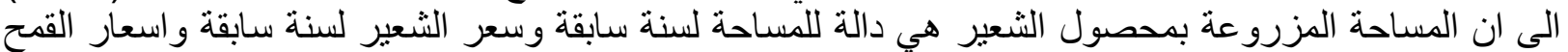

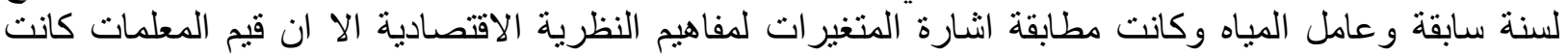

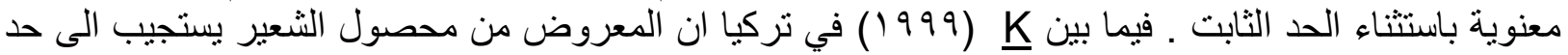

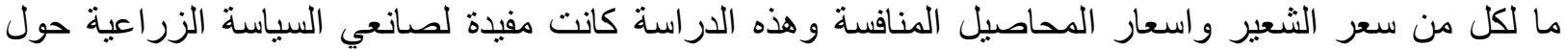

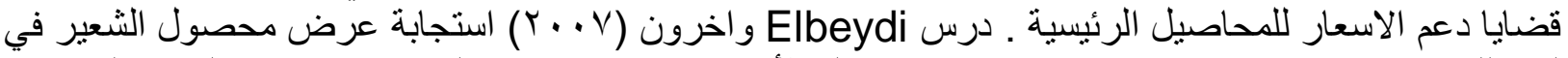

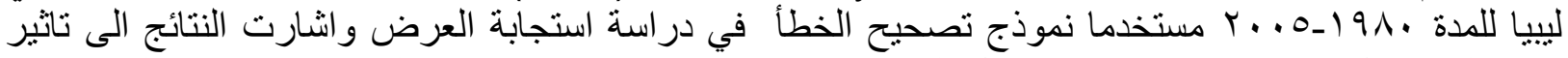

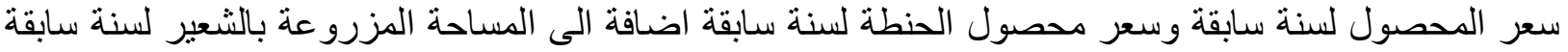

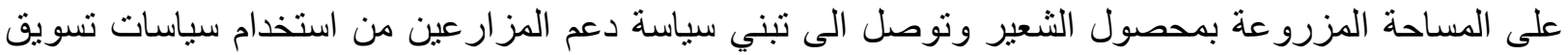

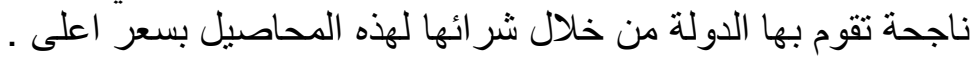

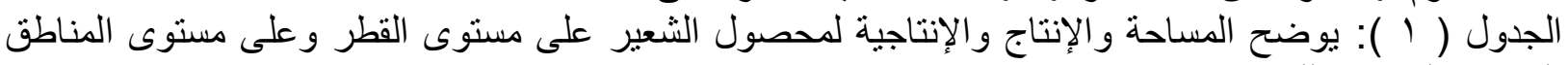

\begin{tabular}{|c|c|c|c|c|c|c|c|c|c|}
\hline \multicolumn{3}{|c|}{ الإنتاجية كغم /دونم } & \multicolumn{3}{|c|}{ الإنتاج .... (طن } & \multicolumn{3}{|c|}{ المساحة المزروعة . . . ا دونم } & \multirow{2}{*}{ السنوات } \\
\hline 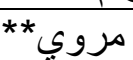 & 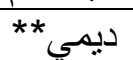 & قطر * ق ق ق & مروي*** & ديمي ** & قطر * ق ق ق & مروي & 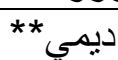 & قطر * ق & \\
\hline rrq & 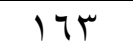 & 194 & מצr & roq & TAr & 1 1 & $r Y \cdot \varepsilon$ & rook & 191. \\
\hline$r \leqslant \Lambda$ & $r / r$ & Yro & $r \leqslant 7$ & ovq & $9 Y 0$ & $1 \% q V$ & $r V \| I$ & $\leqslant 1 . \wedge$ & 1911 \\
\hline Y01 & 187 & $19 V$ & T17 & ONV & $q . r$ & $1 Y 00$ & גזrT & $\varepsilon 0 \wedge r$ & $19 \wedge r$ \\
\hline 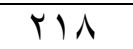 & 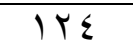 & 104 & rov & $\varepsilon \vee q$ & NTr & $17 \varepsilon 1$ & ГАтт & $00 . \mathrm{T}$ & $19 \wedge r$ \\
\hline YYY & YI & 10 & rq9 & NKG & $\varepsilon \wedge Y$ & $1 V 9 V$ & $r q \leqslant V$ & OTNY & $19 \wedge \varepsilon$ \\
\hline TYY & TrE & TrY & ₹9 & 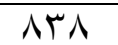 & | & YYII & roß & $O V \leqslant Y$ & 1910 \\
\hline$r \cdot r$ & 100 & $I V T$ & $\varepsilon r$. & 717 & $1 . \leqslant 7$ & YIYY & rqA & 7.5. & 1917 \\
\hline rr. & $V Y$ & IYA & 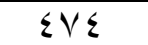 & r7q & $V \varepsilon r$ & r. 70 & rVoA & OAYT & $19 \wedge V$ \\
\hline 174 & r9o & $r \leqslant 0$ & r47 & 1.81 & $1 \leqslant r V$ & $Y Y \leqslant V$ & TrTr & $0 \wedge V \varepsilon$ & 1911 \\
\hline 19. & 71 & 1.7 & $\varepsilon \cdot V$ & YOV & Tז & $r \cdot \Lambda r$ & $\leqslant 190$ & TYVV & 1919 \\
\hline$r \leqslant \Lambda$ & TYO & $r r \xi$ & $T \leqslant Y$ & $M K I Y$ & $110 \leqslant$ & roqr & or^ᄉ & VqY7 & 199 \\
\hline $1 \cdot \varepsilon$ & 77 & $\Lambda$. & roq & $\varepsilon .9$ & 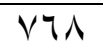 & $r \leqslant Y r$ & $719 \mathrm{~V}$ & 9711 & 1991 \\
\hline roo & $1 \leqslant 0$ & 194 & V7T & $0 \wedge \Lambda$ & $1 T 0 \leqslant$ & rq^q & $\varepsilon .7 V$ & $v .07$ & 1994 \\
\hline $17 \varepsilon$ & $1 Y \Lambda$ & $1 \leqslant 1$ & TVY & 019 & 19. & YYYA & $\varepsilon .70$ & אזTז & 1994 \\
\hline $1 V V$ & $1 \leq Y$ & $10 \leqslant$ & TYA & OYT & 10 & INOY & $r V \cdot r$ & $000 \leqslant$ & $199 \varepsilon$ \\
\hline
\end{tabular}


$\begin{array}{ll}\text { ISSN:2224-9796(Online) } & \text { ISSN: 1815-316X(Print) }\end{array}$

مجلة زر اعة الر افدين

\begin{tabular}{|c|c|c|c|c|c|c|c|c|c|}
\hline $1 \wedge r$ & $1 \pi$ & $10 Y$ & TrV & ए^o & $V I Y$ & IVAT & $r \wedge \wedge 0$ & $\{7 V 1$ & 1990 \\
\hline 197 & IYA & 100 & Tro & TrT & $T \leqslant V$ & $17 \leqslant 1$ & TOY & ¿17 & 1997 \\
\hline $1 \leqslant 9$ & $\wedge 1$ & 1.1 & TMT & 191 & $\varepsilon r$. & 1009 & $r \varepsilon \varepsilon$. & r999 & 1998 \\
\hline TYE & 11. & 101 & TrMT & rqV & $\pi$. & $1 \leqslant \wedge \mu$ & r79V & §1^. & 1991 \\
\hline$r \cdot 1$ & 1 & 71 & rᄉ. & 2.7 & YAT & $1 \% \wedge q$ & rVqr & $\sum \backslash \wedge$ & 1999 \\
\hline 189 & T & $\lambda \varepsilon$ & 19. & $r_{60}$ & 194 & $1.7 \varepsilon$ & $1 \leqslant 1 V$ & rT.. & $r \ldots$ \\
\hline rrV & $r .7$ & MYY & 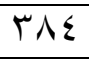 & rrq & $V I T$ & 1119 & 1.10 & TYIS & $r \ldots l$ \\
\hline$r r q$ & $Y \cdot V$ & Y17 & r70 & 079 & ATr & 11.9 & YVOT & T人TY & $r \ldots r$ \\
\hline YOS & IVA & $r \cdot r$ & $r \leqslant T$ & 019 & ᄉт. & $1 T \leqslant 0$ & $r q .1$ & ETOr & $r . . r$ \\
\hline r70 & $I V Y$ & $r 1$. & $\Sigma r^{\prime}$ & r^o & $1.0, \varepsilon$ & 1010 & $r Y \leqslant T$ & rNYq & $r \ldots \varepsilon$ \\
\hline rqA & $1 . r$ & $I V V$ & $\left.\sum \wedge\right\rceil$ & r7q & $V 0 \varepsilon_{6} \leqslant$ & אד 1 1 & 4719 & EYOY & $r \ldots o$ \\
\hline$\Gamma \cdot \Lambda$ & $1 V$. & TYS & 597 & 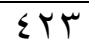 & 91961 & $171 \%$ & $r \leqslant q 1$ & $\varepsilon 1 . r$ & $r \ldots T$ \\
\hline$r \cdot I$ & 19 & $1 V 1$ & 01. & $r r q$ & $V \leqslant \Lambda_{6} r$ & 1794 & rฯAr & $\varepsilon r V \varepsilon$ & $r \ldots v$ \\
\hline TYM & - & Vo & $\varepsilon \cdot \varepsilon$ & - & $\varepsilon \cdot \varepsilon$ & $1 \wedge 1$. & 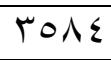 & $049 \varepsilon$ & $r \ldots \Lambda$ \\
\hline TIV & - & $I V A$ & $0 . r$ & - & $0 . r$ & 1014 & אسץ & rAlo & $r \ldots q$ \\
\hline TYV & 119 & 171 & $r q 7$ & $\varepsilon \varepsilon$. & $\Lambda \cdot V$ & 1891 & M170 & $\leqslant q \leqslant Y$ & المتوسط \\
\hline
\end{tabular}

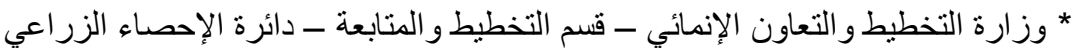

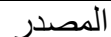

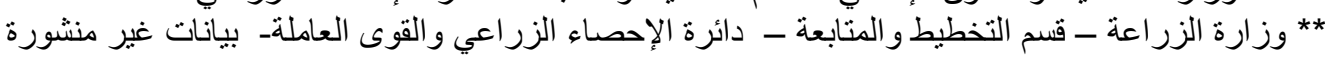

الجدول ( ץ): الأهمية النسبية للمساحة والإنتاج لمحصول الثُعير في المناطق الديمية والمروية ومساهمتها نسبة

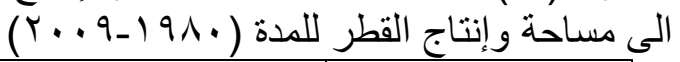

\begin{tabular}{|c|c|c|c|c|}
\hline \multicolumn{2}{|c|}{ المنطقة المروية } & \multicolumn{2}{|c|}{ المنطقة الديمبة } & \multirow[b]{2}{*}{ السنوات } \\
\hline الأهمية النسبية & اللأهمية النسبية & الأهمبة النسبية & اللأهمبة النسبية & \\
\hline $00, r$ & $r v, \cdot$ & $\varepsilon \varepsilon, V$ & 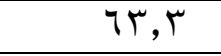 & المتو سط \\
\hline
\end{tabular}

المصدر : احتسبت من قبل الباحثين بالاعتماد على جدول ( )

الجدول (r) :معدلات النمو و الرقم القياسي للتغاير في المساحة والإنتاج والإنتاجية لمحصول القمح في القطر و على مستوى المنطقة الديمية والمروية

\begin{tabular}{|c|c|c|c|c|c|c|}
\hline \multicolumn{2}{|c|}{ المروي } & \multicolumn{2}{|c|}{ الديمي } & \multicolumn{2}{|c|}{ القطر } & \\
\hline اللتنغاير القياسي & السنوي \% معل النمو & للتغاير الرقي & السنوي \% معل النمو & اللتغاير & السنوي معدل النمو & \\
\hline$\cdot, \cdots v$ & $\vee, \Sigma$. & $\cdot, \cdots 7$ & r,o.- & $\bullet, \cdots \varepsilon$ & $\cdot, 7$. & المساحة \\
\hline$\cdot, \cdots V$ & $9, Y$. & $\cdot, \cdot r$ & r,q.- & $\because, \cdots V$ & $r, 0$. & الإنتاج \\
\hline,,$\cdots 0$ & $T, V$ &,,$\cdots$ & $\cdot, 9 \cdot-$ & $\cdot, \cdots 7$ & ץ,乏. & الإنتاجية \\
\hline
\end{tabular}

المصدر: احتسبت من قبل الباحثين بالاعتماد على جدول (0) .

\section{مواد البحث وطرائقه}

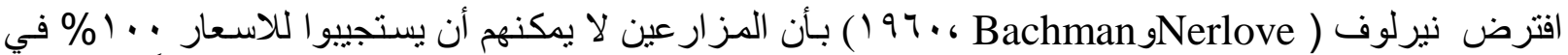

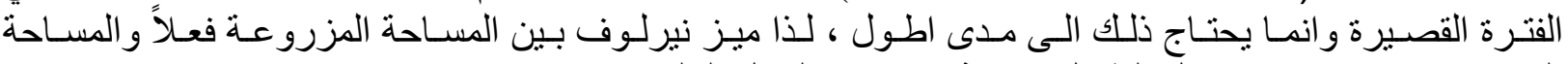
$A t^{*}=b_{0}+b_{1} P_{t-1}+U t$

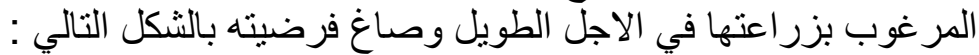




$$
\text { ～ISSN:2224-9796(Online) }
$$

وبين ان المزارع لا يستجيب فوراً للتغيير في السعر و انما تدريجياً فالمزارع بزيد المساحة المزروعة فعلاً و المساحة المطلوب زر زر اعتها وبالصيغة ادناه : $A_{t}-A_{t-1}=B\left(A_{t}^{*}-A_{t-1}\right)$

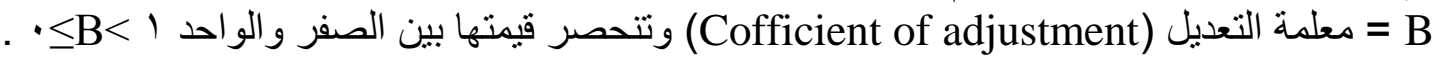

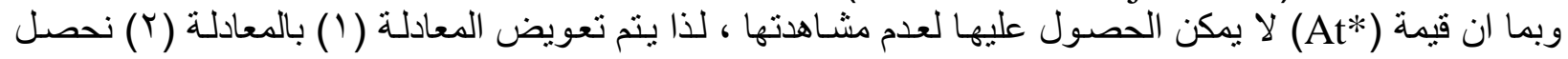

$$
\begin{aligned}
& A t-A_{t-1}=B\left(b_{0}+b_{1} P_{t-1}+U t-A_{t-1}\right) \\
& A t-A_{t-1}=B b_{0}+B b_{1} P_{t-1}+B u t-B A_{t-1} \\
& A t=B b_{0}+B b_{1} P_{t-1}+A_{t-1}-B_{t-1}+B u t \\
& A t=B b_{0}+B b_{1} P_{t-1}+A_{t-1}(1-B)+B u t \\
& A t=C_{0}+C_{1} P_{t-1}+C_{2} A_{t-1}+U t \ldots \ldots \ldots \ldots
\end{aligned}
$$

$\mathrm{C}_{0}=\mathrm{Bb}_{0}, \mathrm{C}_{1}=\mathrm{Bb}_{1}, \mathrm{C}_{2}=(1-\mathrm{B}), \mathrm{Ut}=\mathrm{But}$

ويمكن احتساب مدة التعديل كالاتي : ( Nerlove و W William ، 1901 ().

$$
(1-\mathrm{B})^{\mathrm{n}} \leq \cdot, \cdot 0
$$

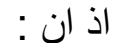

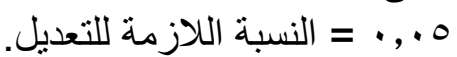
= = ع عدد السنوات اللازمة للتعديل. = B معلمة التعديل.

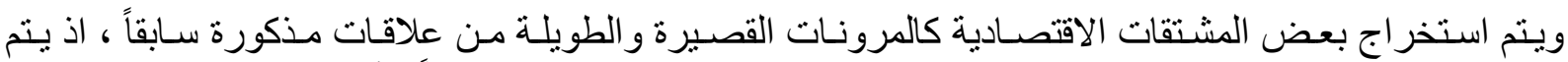

الحصول على مرونة العرض السعرية قصيرة الاجل للمساحة بالطريقة الاعتيادية وفقاً للأنموذج الخطي الاتي : $\varepsilon \varepsilon_{s r}=\frac{\partial A t}{\partial P_{t-1}} \cdot \frac{P s_{t-1}}{A t}$

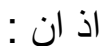

Esr At PS = المتوسط الحسابي البسيط للسعر. $\varepsilon \varepsilon_{L r}=\frac{\varepsilon \varepsilon_{s r}}{B}$

ويتم استخر اج مرونة الاجل الطويل (عLr) بقسمة المرونة قصيرة الاجل على معلمة التعديل أي ان :

يصلح الاطار النظري الذي ذكر سابقا لتوصيف المساحات المزرو عة بالثعير في المنطقتين المروية والديمية وعلى مستوى العراق وعليه فأن النموذج يتضمن لتوصن العلاقات الاتية :

$\ln \mathrm{AB}=\mathrm{bo}+\mathrm{b} 1 \ln \mathrm{PB} \mathrm{t}-1+\mathrm{b} 2 \ln \mathrm{PWt}-1+\mathrm{b} 3 \mathrm{ABt}-1+\mathrm{b} 4 \ln \mathrm{wt}+\mathrm{b} 5 \ln \mathrm{Ra}$

$\ln \mathrm{AB}=\mathrm{bo}+\mathrm{b} 1 \ln \mathrm{PB} \mathrm{t}-1+\mathrm{b} 2 \ln \mathrm{Pvt}-1+\mathrm{b} 3 \ln \mathrm{ABt}-1+\mathrm{b} 4 \ln \mathrm{Ra}+\mathrm{b} 6 \ln \mathrm{Rn}$ $\ln \mathrm{AB}=\mathrm{bo}+\mathrm{b} 1 \ln \mathrm{PB} \mathrm{t}-1+\mathrm{b} 2 \mathrm{kt}-1+\mathrm{b} 3 \ln \mathrm{ABt}-1+\mathrm{b} 4 \ln \mathrm{Ra}+\mathrm{b} 5 \ln \mathrm{Rn}$

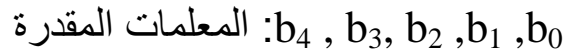

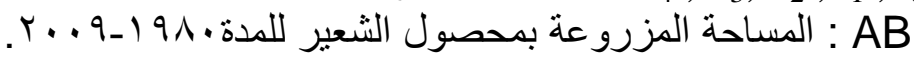

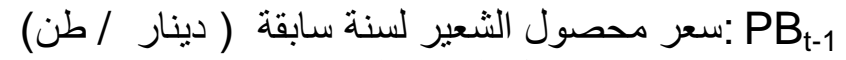

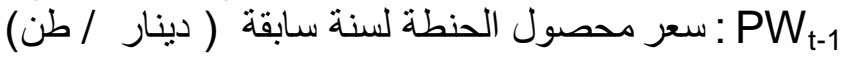
PKt-1

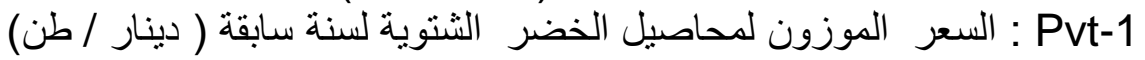




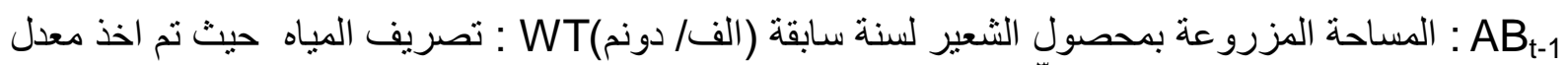
تصريف مياه نهري دجلة والفرات (مُ"اثا) الامطار : RN $\mathrm{RISK}=\left(\mathrm{PB}_{(-1)}-\mathrm{mat}\right)^{2} / \mathrm{mat}$ المخاطرة الانتاجية التي تمثل الانحر اف عن الانتاج حسب المعادلة التالية : PR : الإل Mat $=0.333\left(\mathrm{~PB}_{(-2)}+\mathrm{PB}_{(-3)}+\mathrm{PB}_{(-4)}\right)$

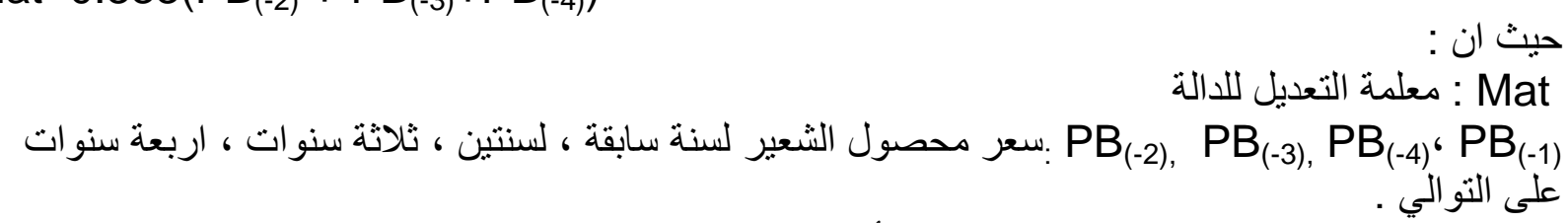

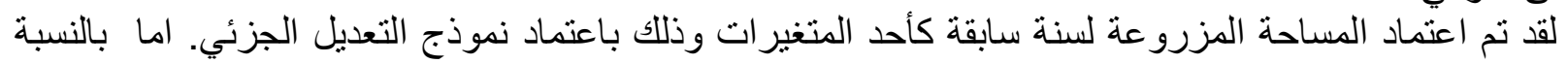

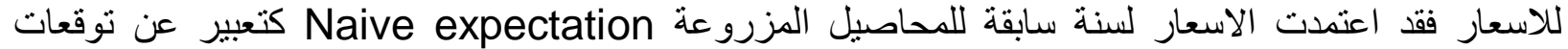

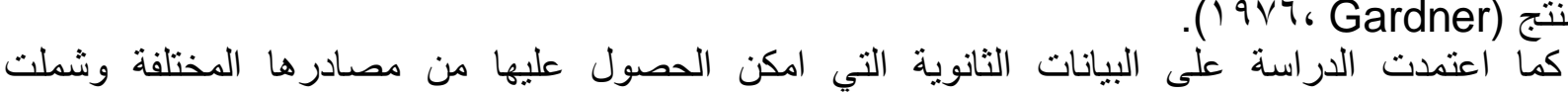

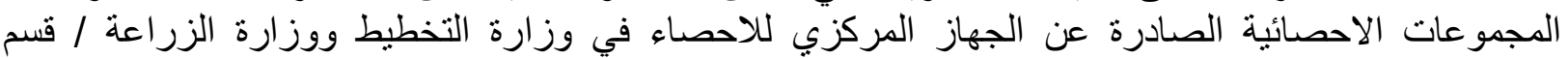

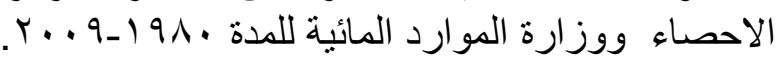

\section{النتائج والمناقشة}

تم تقدير معادلات استجابة عرض المساحة المزروعة بمحصول الشعير في المنطقة المروية والمنطقة الديمية

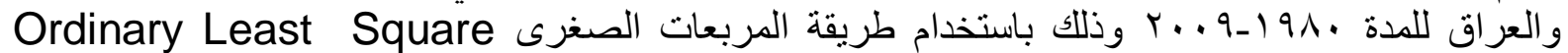

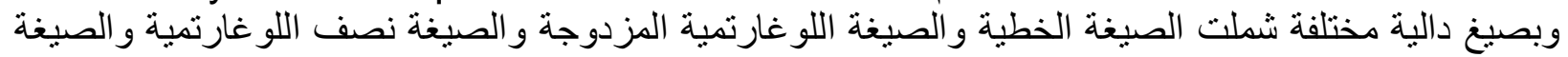

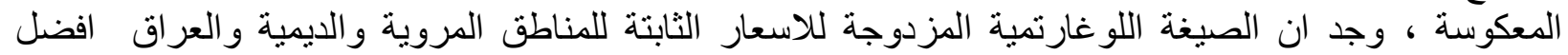

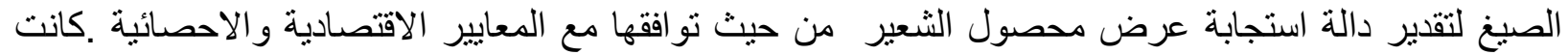

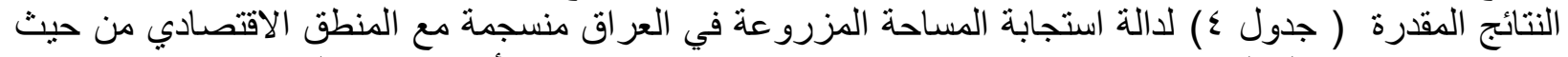

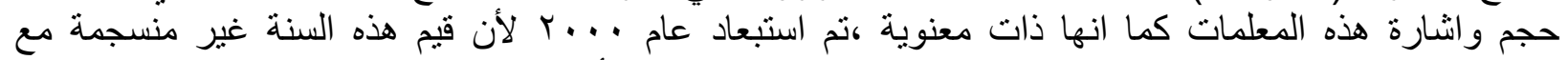

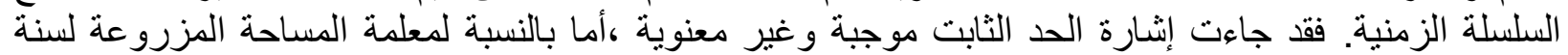

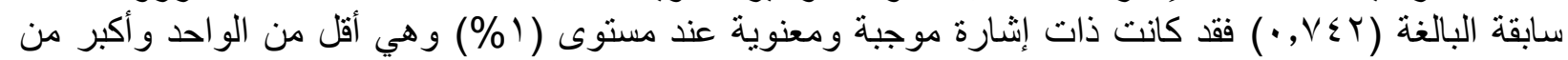

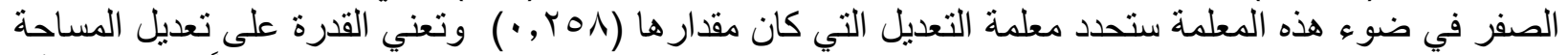

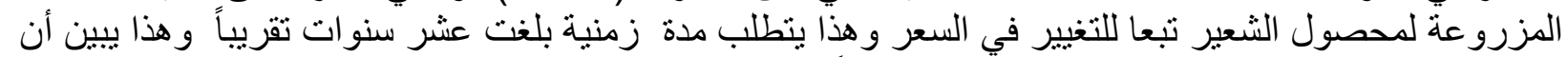

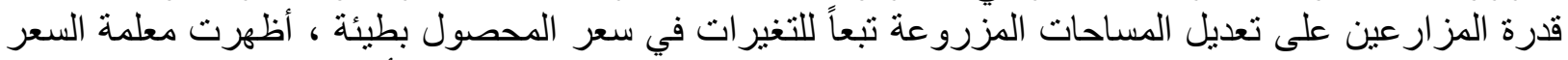

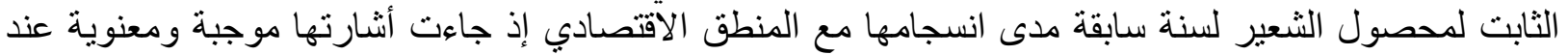

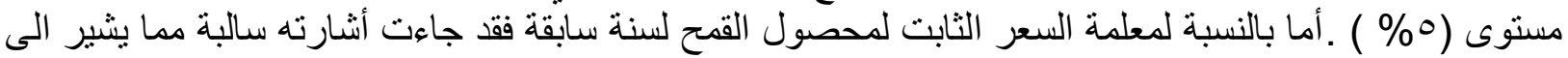

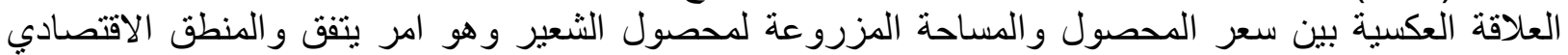

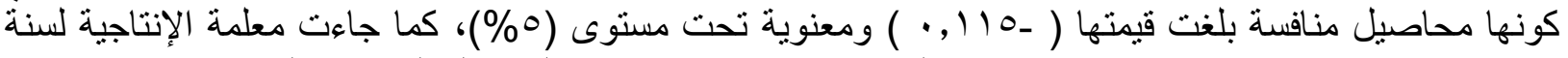

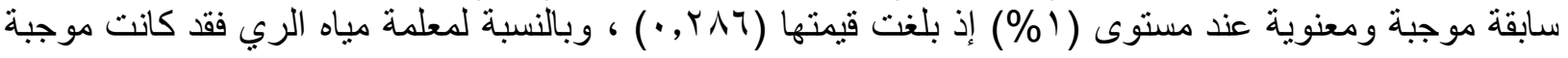

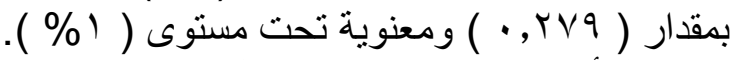

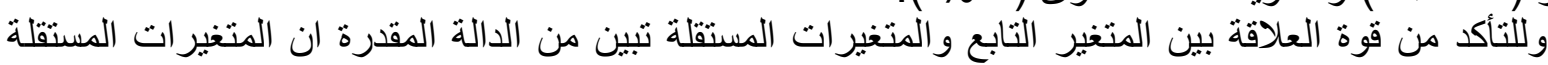

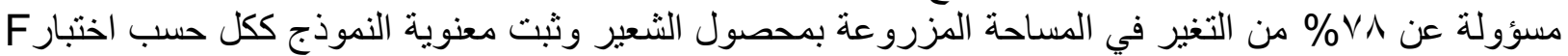

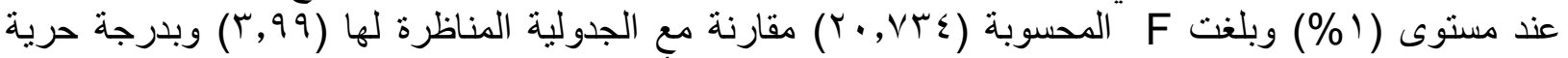

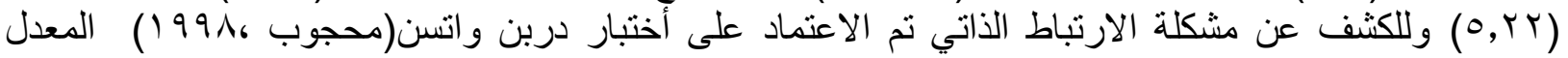

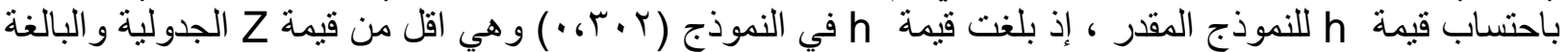

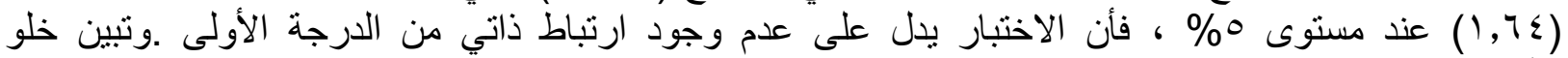

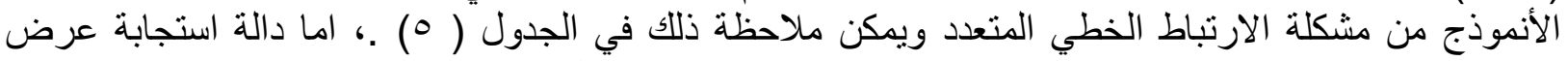

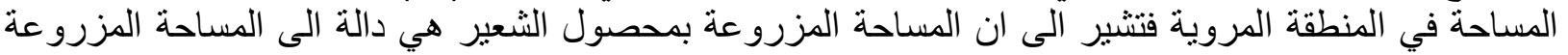

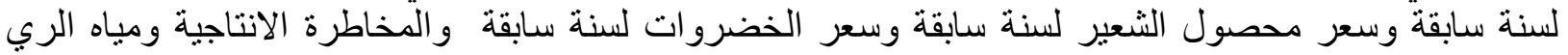

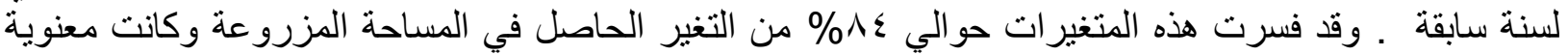

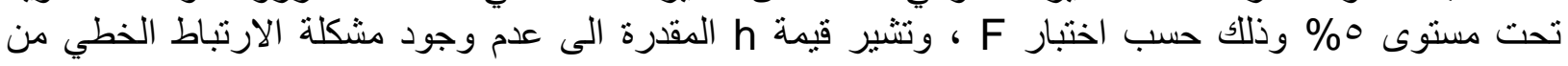




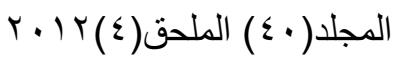 \\ ISSN:2224-9796(Online) \\ ISSN: 1815-316X(Print)}

مجلة زر اعة الر افدين

الدرجة الاولى .جدول ( ب) يتفق التقدير الخاص بمعلمة سعر محصول الثعير لسنة سابقة والمنطق الاقتصادي اذ

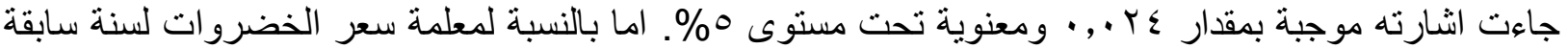

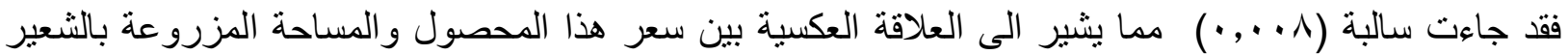
و هو امر يتفق والمنطق الاقتصادي كونها محاصيل منافسة. اما معلمة المساحة المزروعة لسنة سابقة فقد بلغت

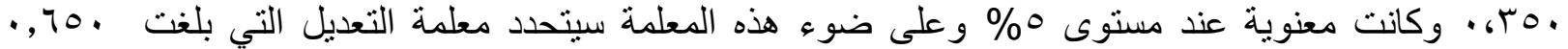

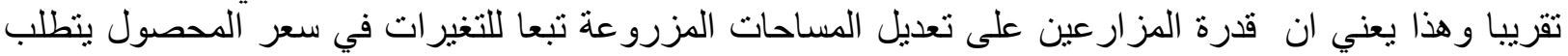

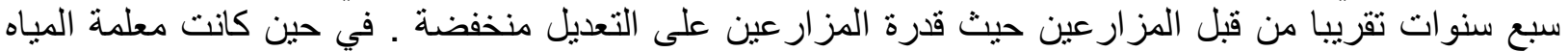

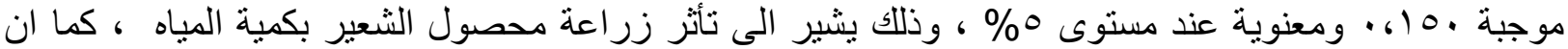

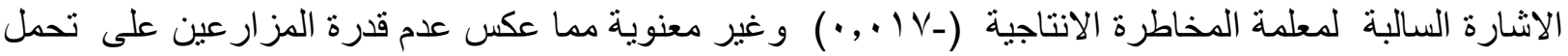

المخاطرة الانتاجية.

تبين من التقدير ات التي تم الحصول عليها للمنطقة الديمية أن معلمة الحد الثابت للأنموذج المقدر ذو أنشارة

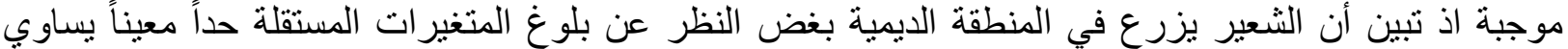

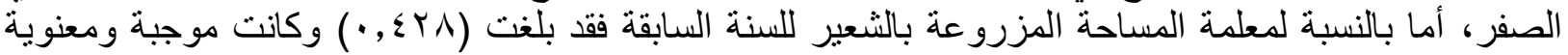

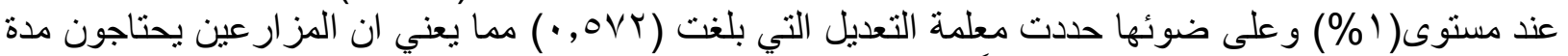

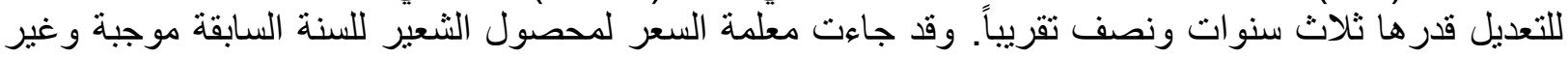

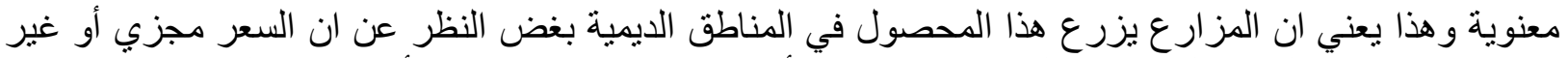

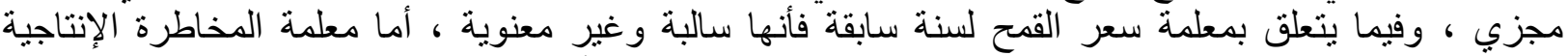

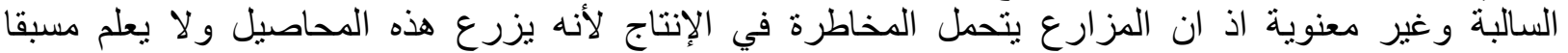

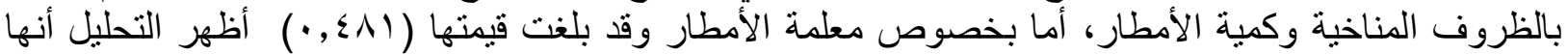

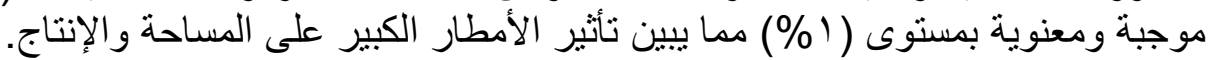

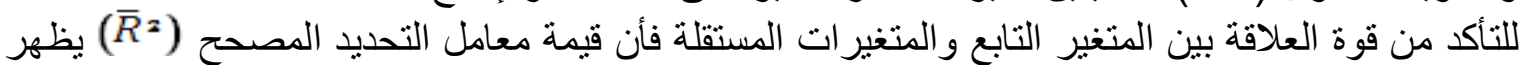

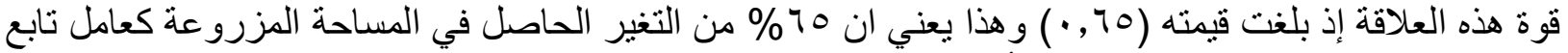

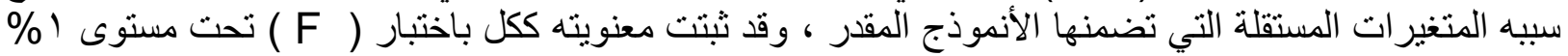

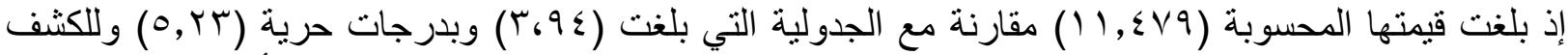

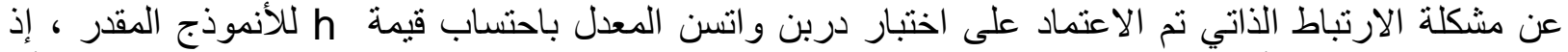

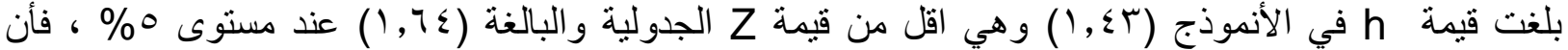

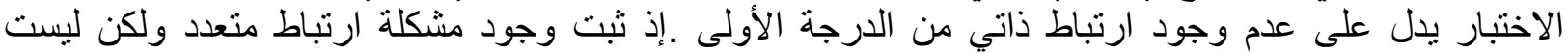

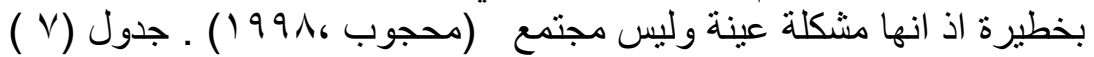
الجدول ( ) ) : تقدير إستجابة عرض محصول الثعير في العراق والمنطقتين الديمية والمروية للمدة

\begin{tabular}{|c|c|c|c|}
\hline في المنابة المتغير التابع الديمية & في المنطقة المتغير التابع & إستجابة المتغير التابع & المتغير ات المستقلة \\
\hline $\begin{array}{l}r, T \cdot r \\
(1, T \vee V)\end{array}$ & $\begin{array}{c}r, \cdot r_{0} \\
(r, \wedge 01) * *\end{array}$ & $\begin{array}{l}\cdot, \cdot 19 \\
(.6 .4)\end{array}$ & الثابت (constant) \\
\hline $\begin{array}{c}\cdot, \leqslant \leqslant q \\
(\mu, \wedge \vee \wedge)^{* *}\end{array}$ & $\begin{array}{c}\cdot, \cdot 70 \\
(\wedge, \leqslant 7 \leqslant)^{* *}\end{array}$ & $\begin{array}{l}.6 V \leqslant r \\
\left(\Lambda_{6} .07\right)^{* *}\end{array}$ & 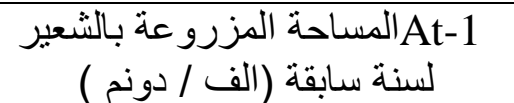 \\
\hline $\begin{array}{c}\cdot, r r q_{-} \\
(r, \cdot \wedge \cdot-)^{*}\end{array}$ & & $\begin{array}{l}. \text { 6)10- } \\
(1, \text { เคน }\end{array}$ & سعر محصول القمح لسنة \\
\hline $\begin{array}{c}\cdot, \cdot r \cdot \\
(\cdot, T r)\end{array}$ & $\begin{array}{l}\cdot, \cdot r \leq \\
(1, \vee q r)^{* *}\end{array}$ & $\begin{array}{c}\cdot, \cdot \leq T \\
(Y, \cdot \cdot T)^{*}\end{array}$ & سعر الشعير لسنة سابقة pbt-1 \\
\hline $\begin{array}{l}\cdot, Y Y \Lambda_{-} \\
\left.(Y, Y V)_{-}\right)^{* *}\end{array}$ & & & سعر البقوليات للسنة السابقة Pkt-1 \\
\hline - & $\begin{array}{l}-\cdot, \cdots \wedge \\
(1,119)\end{array}$ & & سعر الخضروات لسنة \\
\hline $\begin{array}{l}(6,00- \\
(., \text { TVT- })\end{array}$ & $\begin{array}{l}(\cdot .9 T 4) \\
-\cdot . \cdot 1 V\end{array}$ & 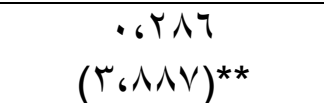 & الإنتاجية (كغم/دونم) \\
\hline
\end{tabular}




\begin{tabular}{|c|c|c|c|}
\hline & $\begin{array}{c}\cdot, 10 \\
(r, \cdot r \varepsilon)^{*}\end{array}$ & $\begin{array}{c}\cdot, Y \vee q \\
(Y, \vee \wedge T)^{* *}\end{array}$ & مياه الري لسنة سابقة (مليار /م") \\
\hline $\begin{array}{c}\cdot, 09 \mu \\
(\varepsilon, 9 r))^{* *}\end{array}$ & - & - & الامطار للسنة الحالية (مليمتر ات) \\
\hline $\begin{array}{l}\cdot, \cdot r \wedge \\
(r, r \wedge q)^{*}\end{array}$ & - & & الزمن \\
\hline • & $\cdot, \wedge \vee$ & $\cdot, \wedge r$ & $\left(R^{2}\right)$ \\
\hline$\cdot, V V$ & $\cdot, \wedge \varepsilon$ & $\cdot, \vee \wedge$ & $\left(\bar{R}^{z}\right)$ \\
\hline$\cdot, \wedge) \leqslant$ & $\cdot, 11$ & $\cdot, r \cdot r$ & قيمة \\
\hline $1 \varepsilon, .0 Y^{* *}$ & $* * Y \vee, Y \vee \wedge$ & $* * Y \cdot, V \leq r$ & (F) قيمة \\
\hline
\end{tabular}

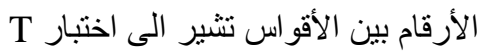

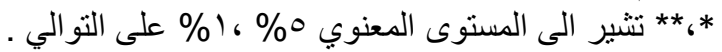
الجدول (0) :مصفوفة الارتباطات البسيطة بين المتغير الثير ات المستقلة لمحصول الثعير على مستوى القطر للمدة

\begin{tabular}{|c|c|c|c|c|c|}
\hline Model & $\mathrm{t}$ & LNQ & LNAT & LNWT & LNP1 \\
\hline $\begin{array}{c}\text { Correlation } \mathrm{t} \\
\text { LNQ } \\
\text { LNAT } \\
\text { LNWT } \\
\text { LNP1 }\end{array}$ & $1, \ldots$ & $\begin{array}{l}., 179- \\
1, \ldots\end{array}$ & 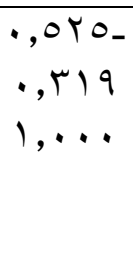 & $\begin{array}{l}\cdot, r \mid q \\
\cdot, \ldots r \\
,, \mid \vee \cdot- \\
1, \ldots\end{array}$ & $\begin{array}{l}\cdot, q \leqslant V_{-} \\
\cdot, r T r \\
\cdot, T r \\
\cdot, \cdot 1- \\
1, \ldots\end{array}$ \\
\hline
\end{tabular}

معامل الارتباط = Y 9 ، .

الجدول(ך) :مصفوفة الارتباطات البسيطة بين المتغيرات المستقلة لمحصول الشعير على المستوى المروي للمدة $(r \cdot .9-19 \Lambda \cdot)$

\begin{tabular}{|c|c|c|c|c|c|}
\hline Model & $\mathrm{t}$ & LNQ & LNAT & LNWT & LNP1 \\
\hline Correlation & $1, \cdots$ &., .91 &., .01 & $\cdot, r 70$ & $\cdot, \leqslant 77$ \\
\hline LNWT & & $1, \ldots$ & • ז", • & $\cdot, 11$. & $-\cdot, 01$ \\
\hline LNRA & & & $1, \ldots$ & $\cdot, 1 \cdot 1$ & ., $\{07$ \\
\hline LNAT & & & & $1, \ldots$ & س.r, \\
\hline LNPE & & & & & $1, \ldots$ \\
\hline LNP1 & & & & & \\
\hline
\end{tabular}

معامل الارتباط = ب 9 , •

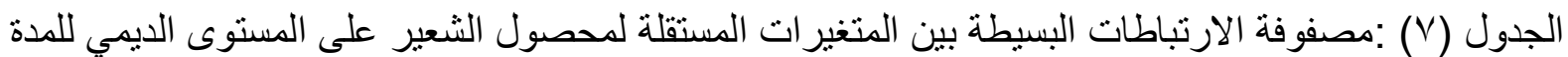

\begin{tabular}{|c|c|c|c|c|c|c|c|}
\hline Model & $\mathrm{t}$ & LNPW & LNRA & LNAT & LNPB & $\frac{(r \cdots q}{\text { LNRN }}$ & $\frac{-191 \cdot)}{\text { LNPK }}$ \\
\hline $\begin{array}{r}\text { Correlation } \mathrm{t} \\
\text { LNPW } \\
\text { LNRA } \\
\text { LNAT } \\
\text { LNPB } \\
\text { LNRN } \\
\text { LNPK }\end{array}$ & $1, \ldots$ & $\begin{array}{l}\cdot, 01 \leqslant- \\
1, \ldots\end{array}$ & $\begin{array}{l}\cdot, r \wedge V- \\
\cdot, \varepsilon \cdot r \\
1, \ldots\end{array}$ & $\begin{array}{l}\cdot, 11 \wedge- \\
., \ldots 0 \\
., 1 \vee \wedge \\
1, \ldots\end{array}$ & $\begin{array}{l}\cdot, r \wedge \cdot- \\
\cdot, 190- \\
\cdot, 1 \Gamma 0 \\
\cdot, 1 \leq r \\
1, \ldots\end{array}$ & $\begin{array}{l}.6 .5 \cdot- \\
.61 Y \wedge \\
.6 Y 09 \\
.61 \leq \varepsilon \\
.6 Y \leq V \\
16 . .\end{array}$ & 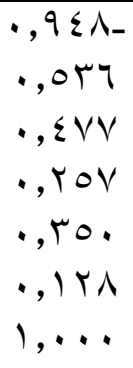 \\
\hline
\end{tabular}




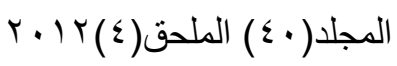
ISSN:2224-9796(Online)
ISSN: 1815-316X(Print)
مجلة زر اعة الر افدين

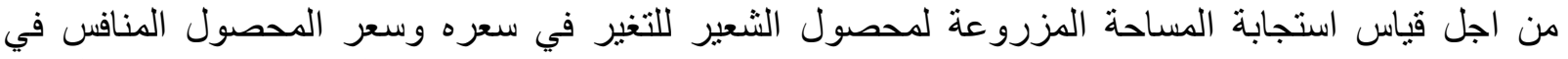
المنطقة المروية والمنطقة الديمية والعراق. احتسبت هذه المرونات عند المتوسطات الحسابية للمتغيرات المعنية

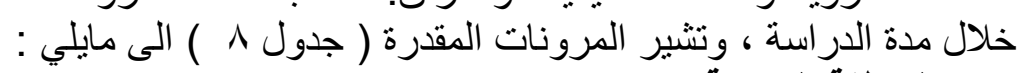

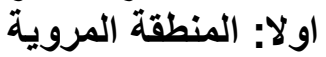

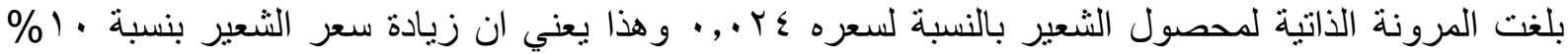

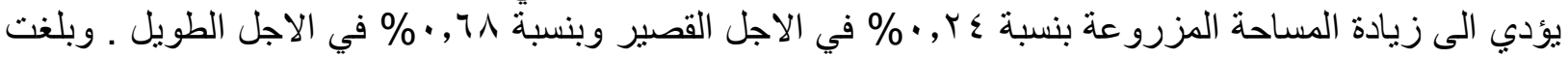

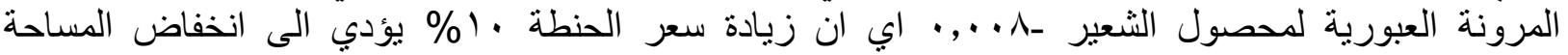

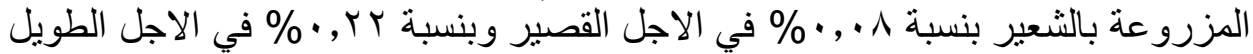

ثانيا: المنطقة الايمية

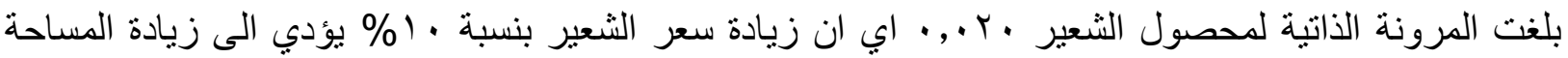

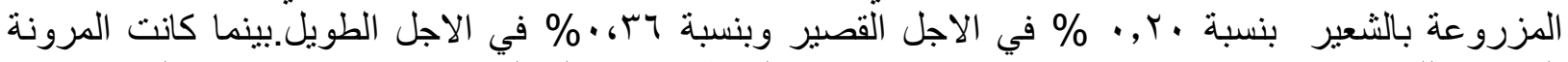

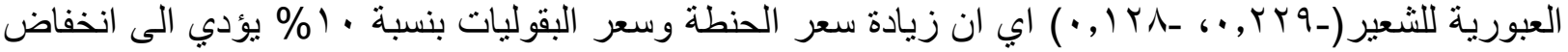

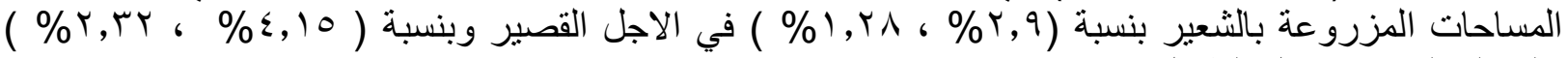

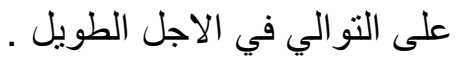

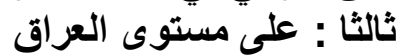

كانت المرونة الذاتية لمحصول العراف الثعير بالنسبة لسعره في العراق اكبر مما كانت علية في المنطقتين المروية

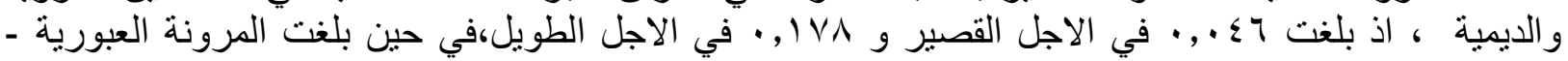

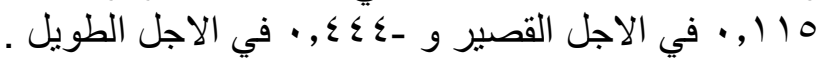

الجدول (^): مرونات الأجل القصير و الأجل الطويل لمحصول الثعير في العراق والمناطق الديمية والمناطق

\begin{tabular}{|c|c|c|c|}
\hline \multicolumn{2}{|c|}{ المرونة العبورية } & المرونة الذاتية & 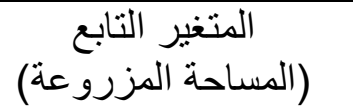 \\
\hline \multicolumn{4}{|r|}{ المساحة على مستوى القطر } \\
\hline \multicolumn{2}{|c|}{$\cdot, 110_{-}$} & $\cdot, \cdot \leq 7$ & الأجل القصير \\
\hline \multicolumn{2}{|c|}{$\cdot, \leqslant \leqslant 0_{-}$} & $\cdot, I \vee \wedge$ & الأجل الطويل \\
\hline \multicolumn{4}{|c|}{ المساحة على مستوى الديمي } \\
\hline$-\cdot, \mid r \wedge$ & •, YYq_ & $\cdot, \cdot r$. & الأجل القصير \\
\hline -•,YMY & $\cdot, \leqslant 10_{-}$ & דוד & الأجل الطويل \\
\hline \multicolumn{4}{|c|}{ المساحة على مستوى المروي } \\
\hline \multicolumn{2}{|c|}{$\cdot, \cdots \Lambda_{-}$} & $\cdot, \cdot r \varepsilon$ & الأجل القصير \\
\hline \multicolumn{2}{|c|}{$\cdot, \cdot, Y_{-}$} & $\cdot, \cdot 71$ & الأجل الطويل \\
\hline
\end{tabular}

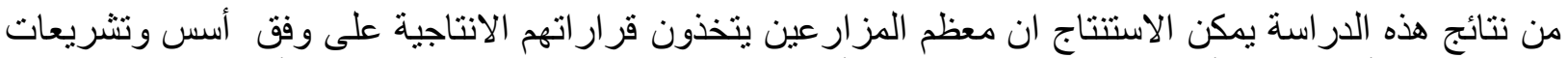

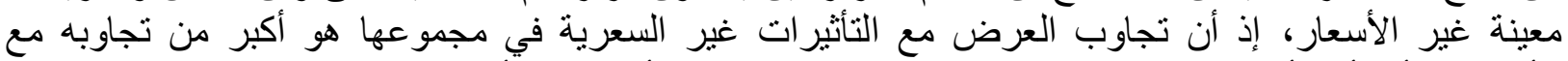

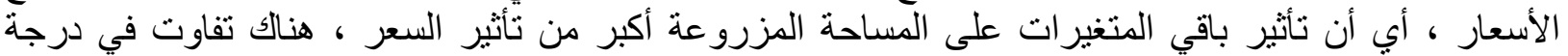

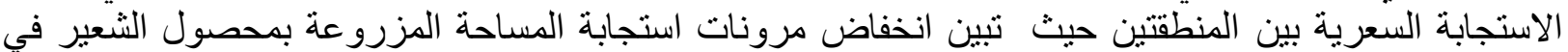

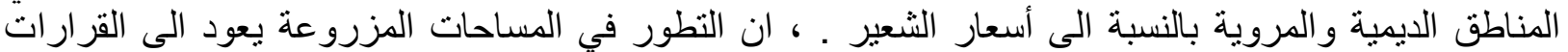

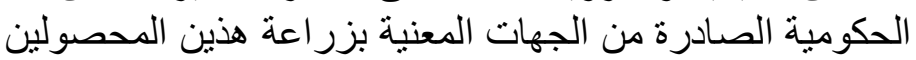

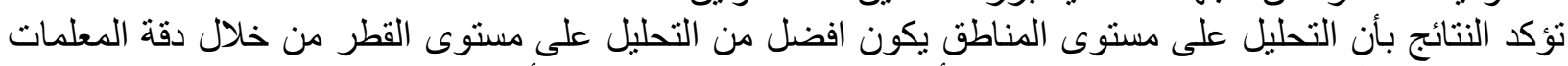
التي تم التوصل اليها بتو افقها مع المنطق الأقتصادي وارتفاع معنويتها الأحصائية وارتفاع قيم المرونات الذيات الذاتية

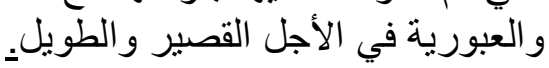




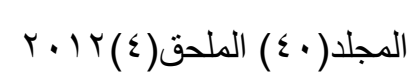

\title{
OF BARLEY SUPPLY RESPONSE IN IRAQ FOR AN ECONOMIC STUDY THE PERIOD 1980-2009
}

Prof. Dr . Muhsen Awaid . Farhan Sarah Ali Hussein Zahra H. Mahmood Department of Agricultural Economics College of Agriculture, University of Baghdad

\begin{abstract}
Barley is one of the main crops in Iraq and is ranked the second most important crop after wheat. This study aimed to estimate supply response of planted acreage for barley in Iraq and irrigated and rained areas for the period from 1980 to 2009 using Nerlove dynamic model. Estimated results indicated that the main variables are lagged price of barley, lagged price of wheat, and lagged planted acreage for barely, besides irrigation water for Iraq and irrigated area, and risk factors of production for rained area .These variables represented about $82 \%, 87 \%$, and $83 \%$ of the change in planted acreage for barley for Iraq, irrigated area, and rained area respectively . The own elasticities of barley with respect to its price for Iraq, irrigated area, and rained area were 0.046, 0.020, and 0.024 respectively. The cross elasticities of barley in respect to wheat price for Iraq and irrigated area were -0.115 and -0.229 respectively. The cross elasticities for barley in respect to legumes for irrigated area were -0.128 and -0.232 in the short-term and longterm respectively, whereas these elasticities in respect to vegetable price were -0.008 and -0.022 in the short-term and long-term respectively.
\end{abstract}

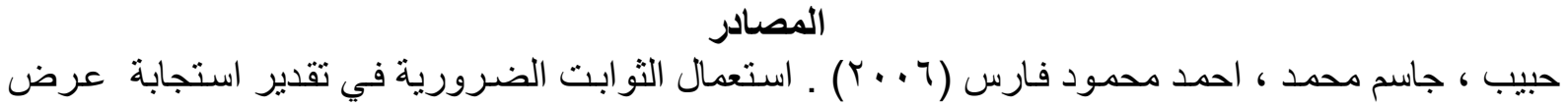

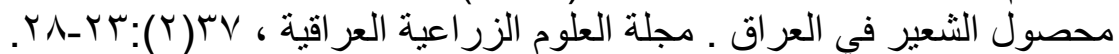

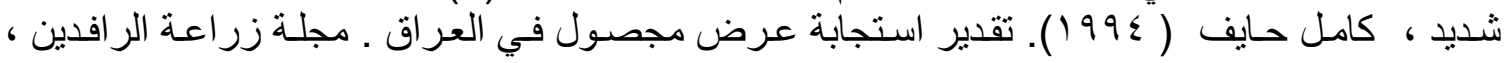

$$
\begin{aligned}
& \text { rq-r): (1) r r }
\end{aligned}
$$

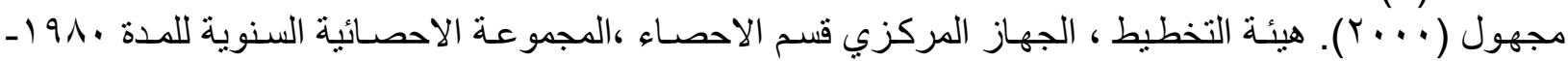

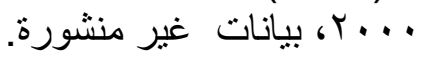

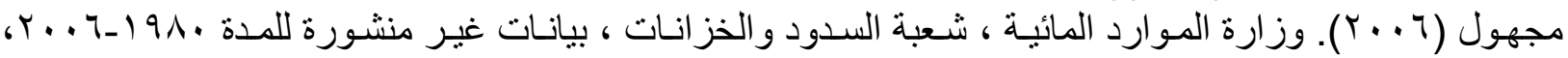

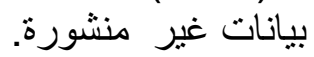

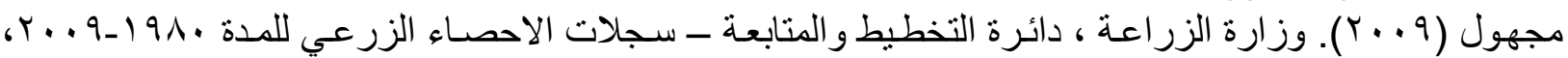

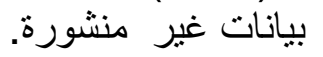

محجوب، عادل عبدالغني (1991). اصول الاقتصاد القباسي: النظرية والتطبيق، الطبعة الاولى، شركة الاعتدال

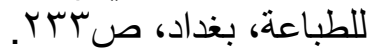

Anderson , K.(1994).Distributed lags and barely acreage response analysis. Aus.J.Agric .Econ.18: 441-446.

Elbeydi, K. R. ; A. A. Aljidi and A. A. Yousef . 2007. Measuring the supply response function of Barley in Libya. Afr. Crop Sci. Conf. Proc. 8:1277-1280.

Gardner , B. L. 1976. Futures prices in supply analysis . Am. J. Agric. Econ. 58:81-85

Koa, A.A.(1999) Acreage allocation of model estimation and policy evaluation for major crops in Turky, Am.J.Agric.Econ.

Mushtaq, K. and P. J. Dawson . 2002. Acreage response in Pakistan : A cointegration approach. Agric. Econ. 27:111-121. 


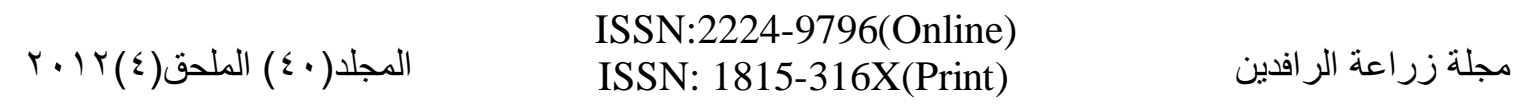

Nerlove, M. (1979). The Dynamics Of Supply: Retrospect and Prospect, Discussion Papers 394, Northwestern University, Center for Mathematical Studies in Economics and Management Science.

Nerlove, M. and K.L. Bachman (1960). The Analysis of Changes in Supply: Problems and Approaches. Journal of Farm Agricultural Economics 42,531-554.

Nerlove , M . and A. William (1958). Statistical estimations of long- run elasticity of supply and demand, J. Farm. Econ .40: 861-881.

Rahji , M. A. Y. ; O. O. Ilmobaya and S. B. Fakayode (2008). Rice supply response in Nigeria : An application of the Nerlovian adjustment model. Nigerian Agric. J. 3:229-234. 